\title{
La biogenèse des mélanosomes
}

\section{L'échiquier de la pigmentation}

> Les mélanocytes situés à la base de l'épiderme produisent des mélanosomes qui sont transférés aux kératinocytes pour assurer la pigmentation de l'épiderme et sa photoprotection contre les rayons ultraviolets. Les mélanosomes, organites apparentés aux lysosomes, sont le lieu de synthèse et de stockage d'un pigment, la mélanine. Leur formation dépend de protéines mélanosomales qui transitent par les voies de biosynthèse et d'endocytose et exploitent les mécanismes moléculaires du trafic intracellulaire. Les acteurs moléculaires impliqués dans le transport des protéines mélanosomales et la biogenèse des mélanosomes sont la cible de mutations dans des maladies génétiques accompagnées d'hypopigmentation comme l'albinisme et les maladies lysosomales. Les études menées sur les mélanocytes issus de souris modèles de ces maladies permettent de comprendre certaines des étapes-clés de la mélanogenèse ainsi que les dysfonctionnements associés à ces pathologies. De plus, décrypter la mélanogenèse facilite également la compréhension d'autres processus physiologiques, comme l'illustrent les similitudes inattendues avec l'amyloïdogenèse dans les maladies neurodégénératives. <

L'épiderme est un épithélium composé majoritairement de kératinocytes et, dans une moindre proportion, de mélanocytes. Ces cellules, pourtant minoritaires, sont de véritables usines dédiées à la synthèse d'un pigment, la mélanine, qui définit la couleur de la peau et des cheveux et représente un système de protection unique contre les radiations ultraviolettes (UV). La gradation de pigmentation de la peau, du blanc au noir, est due à la nature et à la quantité de mélanine produite par les mélanosomes. Généralement, la mélanine se compose de biopolymères d'eumélanine (noire et brune) et de phéomélanine (rouge et jaune). La réaction chimique responsable de la synthèse des différents types de pigment fait intervenir au moins trois enzymes trans-

Cédric Delevoye, Francesca Giordano, Guillaume van Niel, Graça Raposo

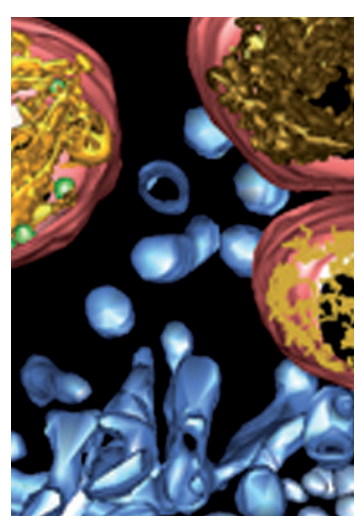

Institut Curie Centre de recherche, CNRS UMR144, 26, rue d'UIm, 75248 Paris, France. graposo@curie.fr cedric.delevoye@curie.fr

membranaires spécifiques des mélanocytes: la tyrosinase, Tyrpl (tyrosinase-related protein 1) et Tyrp2 ou DCT (DOPAchrome tautomerase) [1]. La tyrosinase catalyse la formation des précurseurs de la phéomélanine et de l'eumélanine, alors que Tyrpl et 2 permettent la synthèse de l'eumélanine (Figure 1). Les mélanosomes composés principalement de phéomélanine (phéomélanosomes), encore aujourd'hui peu caractérisés, diffèrent en structure et en composition des eumélanosomes et ne seront pas présentés dans cette synthèse. La séquestration du pigment à l'intérieur des mélanosomes protège le mélanocyte des résidus oxygénés libérés lors de la synthèse et du transfert du pigment. Une fois synthétisée, la mélanine est transférée du mélanocyte au kératinocyte (Figure 1). Le mécanisme de ce transfert reste mal compris et sera discuté ci-après. Les propriétés optiques de la mélanine, qui absorbe les rayons de longueurs d'onde des UV jusqu'au domaine du visible, lui permettent de protéger l'ADN des kératinocytes des effets mutagènes des rayons UV. Dès lors, il apparaît essentiel de comprendre comment les mélanocytes parviennent à fabriquer ces organites hautement spécialisés.

\section{La biogenèse des mélanosomes}

Au début des années 1960, les observations en microscopie électronique ont permis d'analyser l'ultrastructure des mélanosomes [2]. Cependant, leur composition protéique et leur mode de biogenèse sont longtemps restés incompris. L’hétérogénéité de la population mélanosomale dans les mélanocytes de la peau explique en partie qu'il soit difficile d'appréhender leur formation et leur origine $[2,3]$. En effet, quatre stades de maturation du mélanosome sont définis sur la 


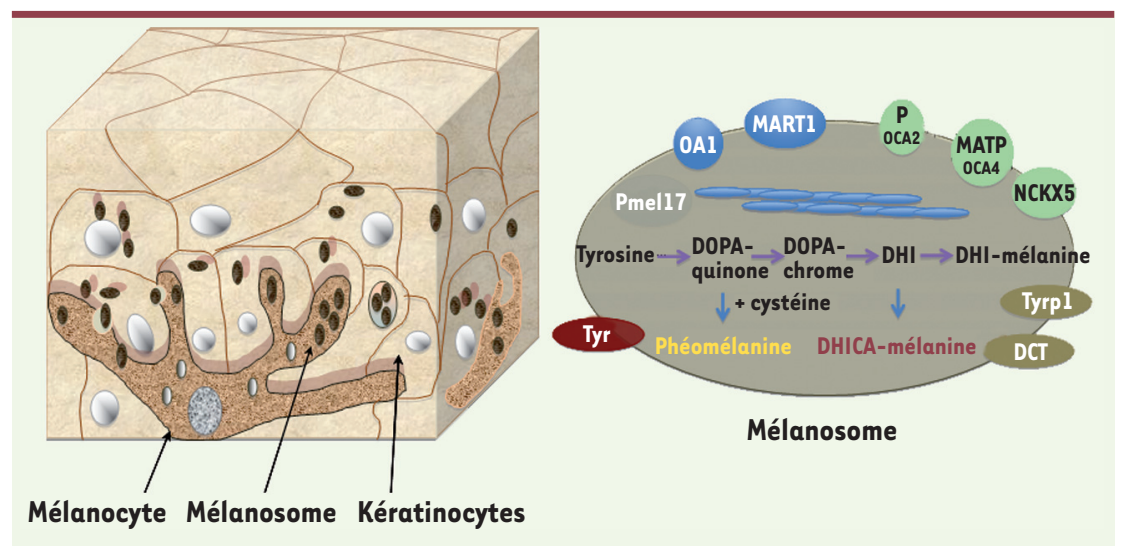

Figure 1. L'unité mélano-épidermique, les protéines mélanosomales et la synthèse de mélanine. Les mélanocytes se localisent à la base de l'épiderme et produisent les mélanosomes. Un mélanocyte peut contacter jusqu'à 40 kératinocytes vers lesquels sont transférés les mélanosomes (gauche). À droite, représentation schématique d'un mélanosome et des protéines mélanosomales ainsi que de la chaîne de biosynthèse de la mélanine (phéomélanine et eumélanine ou DHICA-mélanine). Pmel17 (aussi appellé GP100) est le constituant majoritaire des fibres (bleues) présen-

tes dans la lumière du mélanosome. Les enzymes, tyrosinase (Tyr), tyrosinase-related protein I (Tyrp1) et DCT (dopachrome tautomérase ou Tyrp2) synthétisent la mélanine à partir de la tyrosine importée dans la lumière du mélanosome. OAl (ocular albinism type 1) est un récepteur à 7 domaines transmembranaires impliqué dans la biogenèse des mélanosomes. MART1 (aussi appelé MelanA) a été identifié comme un antigène tumoral mais sa fonction est encore mal comprise. P, dont la mutation cause des albinismes oculocutanés (OCA2), MATP (OCA4), NCKX5 (ou SLC24A5) sont des transporteurs d'anions et de cations et pourraient réguler le pH de la lumière du mélanosome.

base de leur morphologie (Figure 2) [4]. La mélanogenèse se déroule en deux étapes majeures qui permettent tout d'abord de former un organite immature dit non pigmenté (prémélanosomes de stades I et II), qui poursuit progressivement sa maturation en mélanosomes pigmentés (dits de stades III et IV) (Figure 2) [4]. Les mélanosomes de stade IV sont ensuite transférés aux kératinocytes des couches supérieures de l'épiderme (Figure 1).

Il a été proposé que les mélanosomes soient issus du réticulum endoplasmique $[5,6]$. Cependant, plusieurs travaux, dont les nôtres, apportent des arguments en faveur de leur origine endosomale [3, 7]. Les mélanosomes appartiennent à la classe des organites apparentés aux lysosomes (ou LRO pour lysosome-related organelles). Ces organites sont présents dans différents types cellulaires, lymphocytes, macrophages, plaquettes et cellules endothéliales. Cette dénomination de LRO provient des caractéristiques qu'ils partagent avec les lysosomes : ils possèdent des protéines lysosomales (protéines de la membrane du lysosome et hydrolases), sont accessibles à la voie d'endocytose, et leur $\mathrm{pH}$ est acide. Cependant, leurs structures et leurs fonctions sont très différentes. Certains LRO possèdent une double fonction, comme les granules cytolytiques des lymphocytes $T$ cytotoxiques qui assurent la dégradation des macromolécules et la lyse des cellules cibles. En revanche, les mélanosomes ont une fonction unique [3]. En effet, dans les mélanocytes, les mélanosomes coexistent avec les lysosomes et chaque organite remplit des fonctions distinctes (Figure 3).

\section{La voie d'endocytose et ses modulations}

Dans toutes les cellules, la voie d'endocytose se compose de réseaux d'organites et de sous-domaines membranaires auxquels sont attribuées des fonctions cellulaires variées et essentielles telles que l'apport de nutriments, le contrôle des voies de signalisation et la dégradation de macromolécules [8]. Placés au carrefour des voies d'endocytose, de biosynthèse et de dégradation, les endosomes en sont la principale plateforme de tri moléculaire. Ils se composent de domaines vacuolaires et tubulo-vésiculaires comprenant les endosomes précoces de tri et de recyclage. Au cours de leur maturation, ces domaines vacuolaires accumulent progressivement des vésicules dans leur lumière par un processus de bourgeonnement à partir de la membrane délimitant le compartiment. Ces domaines vacuolaires, nommés à ce stade CMV (corps multivésiculaires) précoces, s'individualisent ensuite et se localisent dans la voie d'endocytose; définis par la présence dans leur lumière de vésicules membranaires d'un diamètre de 40 à $80 \mathrm{~nm}$, ces CMV fusionnent avec les lysosomes afin d'y dégrader leur contenu (Figure 3). Dans certains types cellulaires, ces CMV peuvent également fusionner avec la surface cellulaire pour sécréter leurs vésicules intraluminales (exosomes) dans le milieu extracellulaire [9].

L'un des défis rencontrés par une cellule spécialisée comme le mélanocyte est de créer un compartiment membranaire aux caractéristiques morphologiques uniques dans lequel des protéines spécifiques vont remplir leur fonction: synthétiser et stocker le pigment. Pour ce faire, le mélanocyte va utiliser à son profit l'extraordinaire plasticité du réseau endosomal. Comment cette cellule spécialisée réussit-elle ce tour de force? Utilise-t-elle des compartiments cellulaires préexistants ou crée-t-elle de nouvelles voies du trafic intracellulaire? Une fois formés, comment ces mélanosomes restent-ils distincts des endosomes et des lysosomes? Par quels mécanismes la cellule 


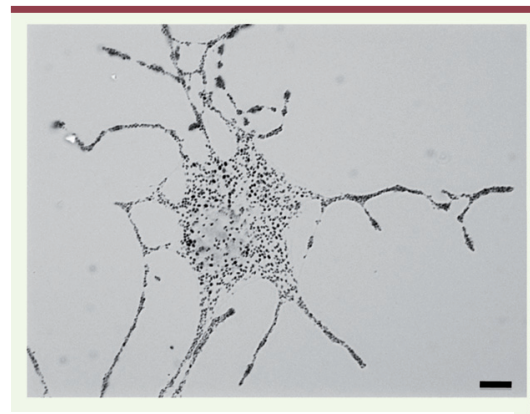

Figure 2. Les mélanocytes et les mélanosomes. Les mélanocytes isolés, observés en microscopie optique (gauche), présentent de nombreuses extensions cellu-

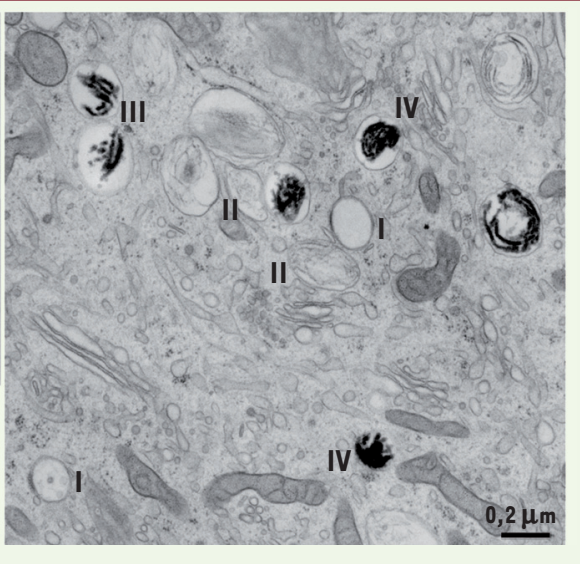

laires (dendrites) où s'accumulent les mélanosomes. Les observations faites par microscopie électronique dans le cytoplasme des mélanocytes (droite) révèlent non seulement des mélanosomes noirs contenant la mélanine (mélanosomes matures), mais également des organites non pigmentés (prémélanosomes, compartiments précurseurs des mélanosomes). Lors de leur formation, les mélanosomes évoluent en 4 stades distincts définis par leur morphologie. Les stades I et II sont blancs, non pigmentés. Ce sont des organites délimités par une membrane et caractérisés respectivement par la présence de vésicules intraluminales membranaires et d'un réseau organisé de structures fibrillaires. Les dépôts de mélanine, noirs en raison de leur densité électronique, sont visibles dans les mélanosomes de stades III et IV. Échelle microscopie optique : $10 \mu \mathrm{m}$. Échelle microscopie électronique : 0,2 $\mu \mathrm{m}$.

réalise-t-elle alors le tri entre les macromolécules à délivrer aux mélanosomes et les protéines résidentes de la voie d'endocytose? Autant de mystères qui, au cours des dernières années, ont commencé à livrer leurs secrets.

\section{L’endosome, site des premières étapes de la mélanogenèse}

La mélanogenèse débute par la formation d'organites non pigmentés ou prémélanosomes (stades I et II). Du fait de leurs morphologie, composition et accessibilité à la voie d'endocytose, les stades les plus précoces (stade I) correspondent à des endosomes communs à la voie d'endocytose et à la biogenèse des mélanosomes [3]. La ségrégation des deux voies a lieu lors de la formation des prémélanosomes de stade II qui, eux, adoptent une forme ellipsoïdale particulière dite en ballon de rugby (Figure 3 ). Cette particularité morphologique est due à la présence de fibres protéiques de nature amyloïde [3, 10] qui servent de support physique à la séquestration de la mélanine. Le constituant principal de ces fibres est une protéine transmembranaire appelée Pmell7 [7]. La polymérisation des domaines luminaux de Pmell7 générés lors de son clivage protéolytique constitue l'élément de base de ces fibres [11]. Ce clivage est effectué par une prohormone convertase de la famille des furines: il sépare le domaine luminal $(M \alpha)$ au caractère fibrillogénétique des domaines transmembranaire et cytoplasmique (M $\beta$ ) [12] (Figure 4 et Encadré 1). Ce clivage a lieu dans les endosomes lors du tri et de la séquestration de Pmel17 sur les vésicules intraluminales des endosomes précoces précurseurs des prémélanosomes (stade I) [13, 14] (Figure 4).

Les mécanismes responsables de l'adressage de Pmel17 aux CMV restent encore mal compris. Conceptuellement, le tri de protéines transmembranaires sur les vésicules internes des CMV nécessite l'action de machineries

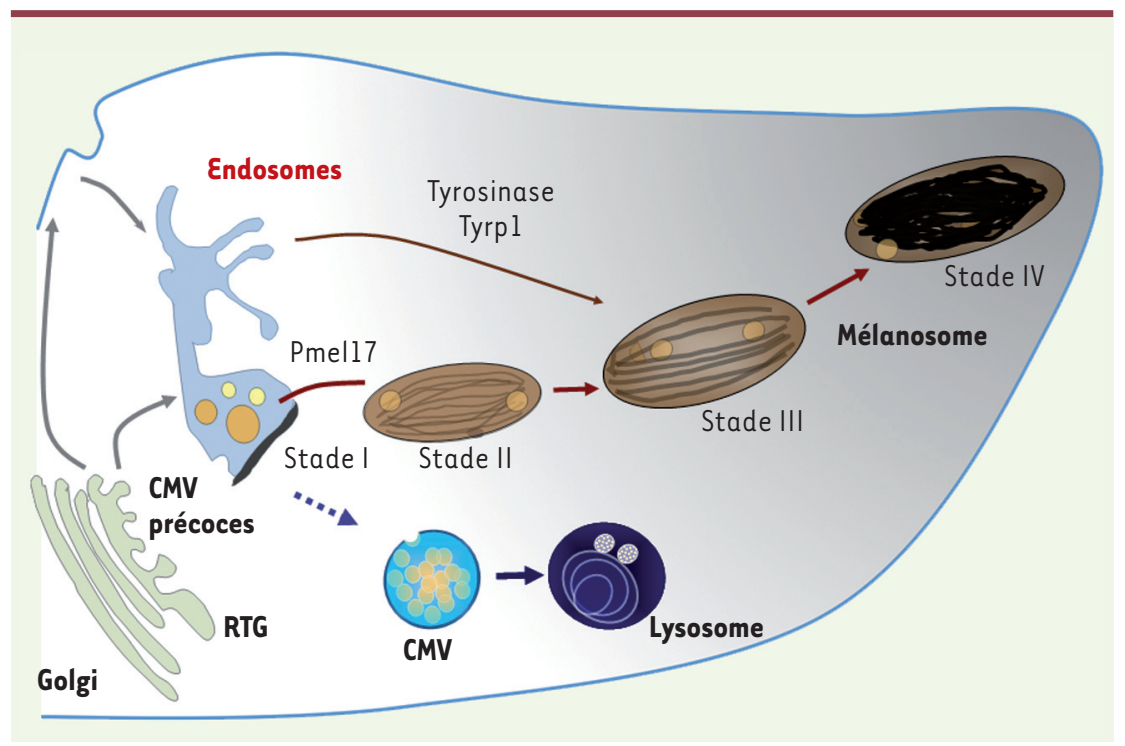

Figure 3. La voie d'endocytose et la biogenèse des mélanosomes. Les mélanosomes proviennent de la voie d'endocytose et restent distincts des lysosomes. La protéine prémélanosomale Pmell7 transite par les endosomes précoces afin de générer les fibres intraluminales caractéristiques des prémélanosomes de stade II. Les enzymes de la mélanogenèse, tyrosinase et Tyrpl, sont adressées depuis des intermédiaires endosomiaux aux mélanosomes en cours de maturation (stades II et III). Les mélanosomes matures, contenant la mélanine (stade IV), sont transportés grâce au réseau de microtubules vers la péripherie de la cellule, dans les extrémités dendritiques, avant d'être transférés aux kératinocytes. CMV : corps multivésiculaires ; RTG : réseau trans-golgien. 


\section{De la prémélanogenèse aux maladies neurodégénératives}

Les fibres protéiques formées par l'autoassemblage du domaine luminal M $\alpha$ de Pmel17 présentent toutes les caractéristiques structurales, biochimiques et biophysiques de fibres amyloïdes pathologiques, lesquelles s'accumulent dans les maladies neurodégénératives telles que l'Alzheimer, les maladies à prions ou le Parkinson [10]. La description récente d'un domaine de Pmel17, nommé CTF (cytoplasmic terminal fragment), issu du clivage du M $\beta$ par une $\gamma$-sécrétase [47], démontre une fois de plus les fortes analogies qui existent entre Pmell7 et les protéines amyloïdogènes comme l'APP (Alzheimer precursor protein). Dès lors, les clivages supplémentaires du domaine $M \alpha$ nécessaires à la formation des fibres pourraient eux aussi dépendre d'enzymes impliquées dans l'amyloïdogenèse [11].

Nos récents travaux ont fait appel à la tomographie électronique pour reconstituer en 3 dimensions les intermédiaires précoces de la mélanogenèse. Ces résultats ouvrent de nouvelles perspectives quant à la compréhension des bases cellulaires de l'amyloïdogenèse (voir [13] et http://www.alzforum.org/new/detail.asp?id=1959). Cette étude montre que les fibres générées lors du clivage de Pmell7 sont formées dans des compartiments endosomiaux où les vésicules intraluminales, du fait de la présence de lipides particuliers dans leur membrane, joueraient un rôle de « nucléateur » des fibres [13] (Figure 4). Ces lipides forment des radeaux ou rafts lipidiques au sein des membranes et favoriseraient la formation de fibres amyloïdes pathologiques [48]. Comme il a été proposé que les endosomes soient impliqués dans l'amyloïdogenèse pathologique, il est nécessaire de poursuivre ces études qui, au-delà de la mise en évidence des mécanismes de la prémélanogenèse, aideront à la compréhension des bases cellulaires et moléculaires des maladies neurodégénératives.

moléculaires qui concentrent spécifiquement un cargo $^{1}$ donné dans un domaine membranaire particulier de la membrane de l'endosome [9]. Ce domaine est ensuite invaginé dans la lumière de l'endosome et forme un bourgeon qui, après scission avec la membrane limitante de l'endosome, devient une vésicule intraluminale. Le mécanisme de tri endosomal le mieux caractérisé nécessite l'action concertée de quatre complexes protéiques appelés ESCRT (endosomal sorting complex required for transport) [15]. De façon inattendue, le tri et la séquestration de Pmell7 dans les vésicules intraluminales des CMV (précurseurs des prémélanosomes) sont indépendants de la machinerie ESCRT [14]. Néanmoins, ce tri est nécessaire au clivage de Pmel17 et à la libération de son domaine luminal qui, dès lors, formera les fibres caractéristiques du prémélanosome et servira de cocon lors de la synthèse du pigment (Figure 4).

\section{La mélanogenèse tardive : un trafic établi grâce aux maladies génétiques}

Les enzymes impliquées dans la synthèse de la mélanine (par exemple : tyrosinase, Tyrpl) sont transportées vers les prémé-

${ }^{1}$ Nous garderons le terme anglais cargo qui désigne les protéines transmembranaires ou non (enzymes ou autres) qui sont véhiculées vers les compartiments cellulaires appropriés via un routage spécifique aux cellules spécialisées (ici les mélanocytes). lanosomes, permettant ainsi la progression de ces derniers vers des stades où ils contiennent du pigment (III et IV) (Figure 3). Plusieurs travaux récents montrent que ces étapes sont altérées dans une maladie génétique autosomale récessive accompagnée d'hypopigmentation, le syndrome d'Hermansky-Pudlak (HPS) (voir Encadré 2) [16]. Le HPS résulte de la mutation de gènes codant pour des protéines qui participent à diverses étapes du trafic intracellulaire.

Pour véhiculer les protéines mélanosomales vers les mélanosomes, la cellule doit mettre en place des systèmes de reconnaissance qui permettent leur identification et leur tri parmi toutes les autres protéines de la cellule. AP-3 (adaptor protein-3) est l'un des quatre complexes adaptateurs hétérotétramériques. II interagit avec des séquences d'acides aminés particulières (signaux di-leucine) situées dans les domaines cytoplasmiques des protéines transmembranaires et permet leur séquestration dans des intermédiaires de transport. Puis, ces derniers sont ciblés vers un compartiment avec lequel ils fusionnent pour y délivrer leur contenu. Dans les mélanocytes, AP-3 interagit avec la tyrosinase et participe à son transport vers les mélanosomes [17]. En effet, dans les mélanocytes issus de patients atteints de HPS2 ou de souris pearl ou mocha (portant une mutation dans une des sous-unités du complexe AP-3) (Figure 5), la tyrosinase s'accumule dans les endosomes [17]. Comme AP-3 se localise dans des vésicules et bourgeons associés aux endosomes précoces, cela suggère que cet adaptateur transporte la tyrosinase des endosomes précoces aux mélanosomes [17]. Cependant, AP-3 n'organise pas la totalité du transport des enzymes de la mélanogenèse car: (1) le pelage des souris pearl ou mocha est coloré [18] ; (2) les mélanocytes de patients HPS2 produisent toujours des mélanosomes d'apparence normale [17] ; (3) la rétention de la tyrosinase dans les endosomes reste partielle car elle se localise dans des vésicules de transport [17]; (4) la Tyrpl, une autre enzyme de la mélanogenèse, n'interagit pas avec AP-3 et son transport aux mélanosomes n'est pas altéré en son absence [17]. Ces observations ont conduit à considérer le possible rôle d'un autre adaptateur moléculaire apparenté à $A P-3$, nommé $A P-1$, qui est intact dans le HPS. De façon intéressante, AP-1 interagit avec les deux enzymes-clés de la mélanogenèse (la tyrosinase et Tyrpl) et prend partiellement en charge le transport de la tyrosinase lorsque AP-3 n'est plus fonctionnel $[17,19]$. De plus, lorsque AP-1 est inactif, Tyrpl s'accumule dans des endosomes précoces suggérant qu'au moins deux enzymes impliquées dans la synthèse de la mélanine transitent par ces plate-formes de tri avant d'atteindre le mélanosome [17, 19]. 


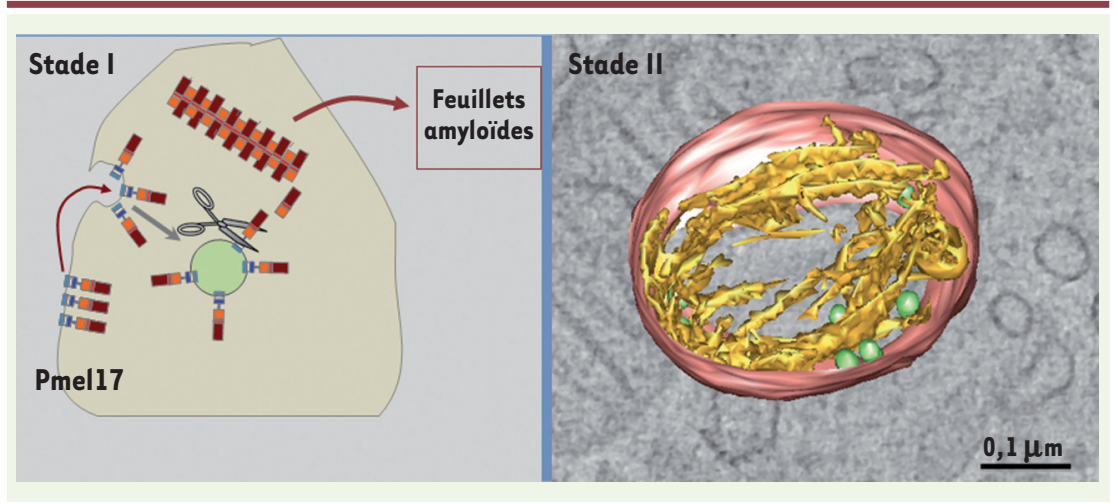

Figure 4. La protéine Pmell7 compose les fibres des prémélanosomes. Pmel 17 est une protéine transmembranaire qui transite par des endosomes (les prémélanosomes de stade I). Dans ces organites, Pmell7 est clivée par une prohormone convertase de la famille des furines ainsi que par d'autres protéases mal caractérisées. Les domaines luminaux de Pmell7 ainsi libérés s'auto-assemblent et forment des fibres qui s'organisent en feuillets amyloïdes (gauche). Cette organisation est mise en évidence par reconstruction 3D en tomographie électronique (droite). Des coupes épaisses $(400 \mathrm{~nm})$ de mélanocytes fixés par congélation à haute pression ont été analysées par microscopie électronique à transmission à $200 \mathrm{kV}$. Des séries d'images ont été acquises avec un logiciel de tomographie (Explore 3D), puis reconstruites (imod) pour établir les modèles 3D correspondants. Le modèle 3D met en évidence des fibrilles (jaune) émanant des vésicules intraluminales (vertes) au sein du prémélanosome de stade II (rouge) (d’après [13]) (échelle : 0,1 $\mu \mathrm{m}$ ).

\section{Le syndrome de Hermansky-Pudlak}

Le syndrome de Hermansky-Pudlak (HPS) se caractérise par un albinisme oculocutané et des troubles associés au dysfonctionnement des différents LRO (défauts de coagulation, fibrose pulmonaire et immunodéficiences). Le HPS résulte de la mutation dans au moins un des huit gènes candidats chez l'homme et des seize gènes chez la souris (Figure 5). Même si les pathologies associées au HPS restent confinées aux types cellulaires qui produisent des LRO (ex. mélanocytes, granules denses des plaquettes), l'expression des gènes mutés dans ces maladies est ubiquitaire. Certaines des protéines codées par ces gènes ont des fonctions déjà décrites (I'adaptateur AP-3, le complexe HOPS, l'enzyme Rab Geranyl geranyl transferase). Au contraire, d'autres protéines n'ont pas de rôle connu, à l'instar de celles qui s'assemblent en des complexes nommés BLOC (biogenesis of lysosome related organelles complex) (Figure 5). À ce jour, les protéines mutées dans le HPS semblent uniquement impliquées dans la formation des stades tardifs de mélanogenèse. Elles régulent le transport endosomal des enzymes de la mélanogenèse et de cargos nécessaires à leur activité enzymatique.

\section{Spécialisation du trafic intracellulaire des mélanocytes}

Dans la majorité des types cellulaires, les protéines cargos triées par AP-1 ou AP-3 sont adressées respectivement aux endosomes précoces et aux endosomes tardifs ou aux lysosomes [20]. En revanche, dans les mélanocytes, les effecteurs doivent s'adapter à ces voies de transport très spécifiques vers les mélanosomes qui concentrent les enzymes de la mélanogenèse. Ici encore, les études issues des mélanocytes de souris modèles du syndrome d'Hermansky-Pudlak permettent de proposer des candidats intervenant dans ces étapes-clés de la mélanogenèse : les complexes BLOC (biogenesis of lysosome related organelles complex). Au nombre de trois, BLOC- $1,-2$ et -3 , ces complexes sont formés de plusieurs protéines (Figure 5). BLOC-1 est composé de 8 sous-unités: les gènes correspondant à deux d'entre elles sont mutés dans le HPS humain (Dysbindin-HPS7 et BLOS3HPS8) et ceux qui correspondent à cinq de ces protéines sont mutés dans des modèles murins du HPS (capuccino, sandy, reduced pigmentation, muted et pallidin) [4]. Les mutations qui affectent le complexe BLOC-1 entraînent les phénotypes d'hypopigmentation les plus sévères rencontrés dans le HPS [16]. Dans les mélanocytes de souris déficientes pour des sous-unités de BLOC-1, la distribution de Tyrpl est très altérée comme le démontre sa rétention massive dans les endosomes. En revanche, la localisation de la tyrosinase n'est que partiellement affectée. Bien que cette enzyme-clé soit encore présente dans les mélanosomes, ces mélanocytes ne synthétisent plus de pigment. L'explication d'une telle absence de pigmentation est venue de la démonstration qu'une altération fonctionnelle du complexe BLOC-1 modifie fortement la distribution d'une protéine essentielle à la fonction de la tyrosinase, le transporteur du cuivre ATP7A (aussi appelé transporteur de Menkes car son gène est muté dans la maladie du même nom). En effet, l'activité enzymatique de la tyrosinase est dépendante de sa capacité à lier les ions cuivre [21]. Le transport d'ATP7A des endosomes aux mélanosomes requiert BLOC-1. La présence d'ATP7A dans les mélanosomes permet le transport du cuivre nécessaire à l'activité de la tyrosinase [22]. Ainsi, les mutations des sous-unités du complexe BLOC-1 altèrent, directement ou non, l'activité de deux enzymes-clés de la mélanogenèse, ce qui se traduit par une hypopigmentation importante [23].

Le destin de Tyrpl est différemment affecté dans les mutants $A P-3$ et $B L O C-1$, et ces deux complexes se localisent dans des domaines endosomiaux distincts $[23,24]$. Au contraire, et au vu des phénotypes engen- 


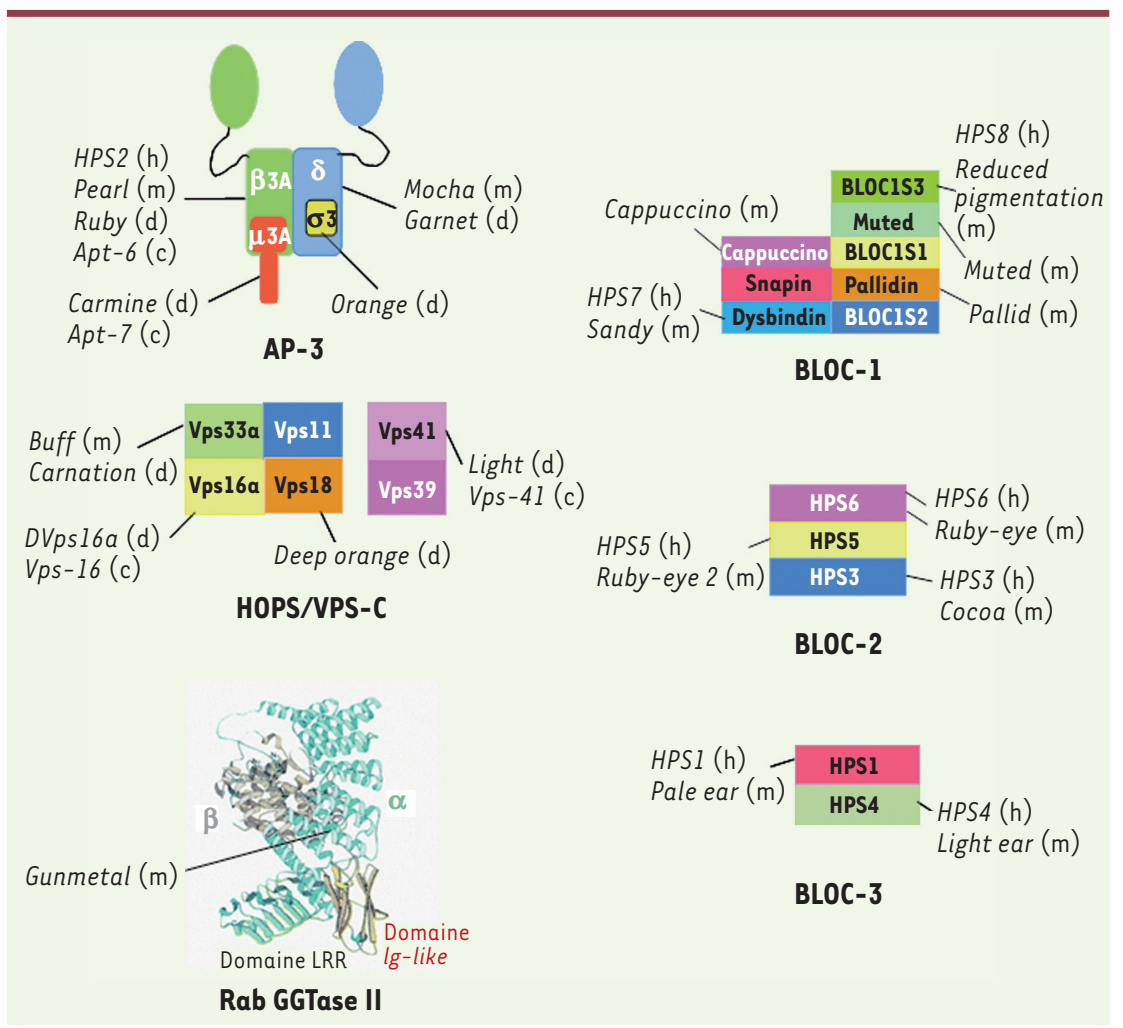

Figure 5. Protéines mutées dans le syndrome d'Hermansky-Pudlak (HPS). Le HPS résulte de la mutation d'au moins un gène parmi huit gènes candidats chez l'homme et huit supplémentaires chez la souris. De plus, les mutations de gènes homologues chez Drosophila melanogaster et Caenorhabditis elegans causent respectivement une malformation des granules pigmentaires de l'œil ou des granules de l'intestin. La quasi-totalité de ces gènes codent pour des sous-unités de complexes protéiques ubiquitaires impliquées dans la biogenèse des LRO. Certains d'entre eux ont une fonction connue dans le trafic intracellulaire. Le complexe AP-3 interagit avec des séquences d'acides aminés présentes dans le domaine cytosolique de protéines transmembranaires. II est impliqué dans leur transport des endosomes vers les lysosomes ou les organites apparentés aux lysosomes. Le complexe HOPS (homotypic protein sorting) interagit avec les protéines SNARE (soluble $\mathrm{N}$-ethylmaleimide sensitive factor [NSF] attachment protein receptors) impliquées dans la fusion membranaire. La géranyl géranyl Rab transférase II (RabGGTase II) catalyse la prénylation de petites GTPases de la famille Rab, une modification nécessaire à leur association aux membranes. Par exemple, une diminution de son activité dans les souris Gunmetal entraîne la localisation cytosolique de certaines Rab et bloquent ainsi des étapes de trafic membranaire. En revanche, les sous-unités des complexes BLOC (biogenesis of lysosome related organelles) BLOC-1, BLOC-2, BLOC-3 ne présentent ni motifs structuraux, ni homologies significatives avec des protéines connues et leurs fonctions restent mal comprises. Cette figure illustre les sous-unités des différents complexes connus chez l'homme (h), la souris ( $\mathrm{m}$ ), la drosophile (d) ou C. elegans (c). Structure de Rab GGTase II : vert, domaine hélical de la sous-unité $\alpha$; jaune : domaine lg-like ; vert en feuillets : domaine LRR (leucine rich repeat); gris : sous-unité $\beta$ (d'après [4]).

drés par leur dysfonctionnement, AP-1 et BLOC-1 pourraient coordonner une seule et même voie, même si cela reste à démontrer $[19,23]$. On peut proposer que ces complexes définissent des voies distinctes mais complémentaires pour rejoindre le mélanosome à partir des endosomes. Au cours de la mélanogenèse, ces routes permettraient de délivrer aux mélanosomes, de manière séquentielle, des protéines ayant des fonctions diverses (enzymes, transporteurs) [24, 25]. De plus, la capacité de ces complexes à interagir avec d'autres machineries moléculaires (moteurs, SNARE [soluble $N$-ethylmaleimidesensitive facteur (NSF) attachement protein receptors]) leur permettrait de réguler diverses étapes du trafic intracellulaire dans le mélanocyte et, par conséquent, la mélanogenèse et la pigmentation du mélanocyte.

\section{Un dialogue étroit requis pour le trafic des endosomes aux mélanosomes}

Une fois le tri des cargos de la mélanogenèse organisé au niveau des endosomes précoces, les intermédiaires de transport enrichis en ces cargos rejoignent le mélanosome pour y être délivrés. AP-3, AP-1 et BLOC-1 trient des cargos de la mélanogenèse dans les membranes endosomales (Figure 6). Alors, quels complexes protéiques permettraient leur adressage aux mélanosomes? BLOC-2 pourrait être un acteur privilégié de ce processus. II se compose de 3 sousunités (HPS3, 5 et 6) [26, 27] mutées chez les patients atteints du HPS et dans des modèles de souris (rubyeye, ruby-eye-2 et cocoa) [16]. L'hypopigmentation observée lorsque BLOC-2 est mutée est moins sévère que celle qui accompagne les mutations de BLOC-1. Elle se caractérise par un défaut de distribution de Tyrpl et, dans une moindre mesure, de la tyrosinase qui s'accumulent dans des vésicules membranaires qui joueraient le rôle d'intermédiaire de transport à l'interface entre les endosomes précoces et les mélanosomes [28]. De plus, BLOC-1 et BLOC- 2 interagissent directement et génétiquement [18, 24], suggérant fortement que ces deux complexes interviennent de manière séquentielle, en triant puis acheminant les 


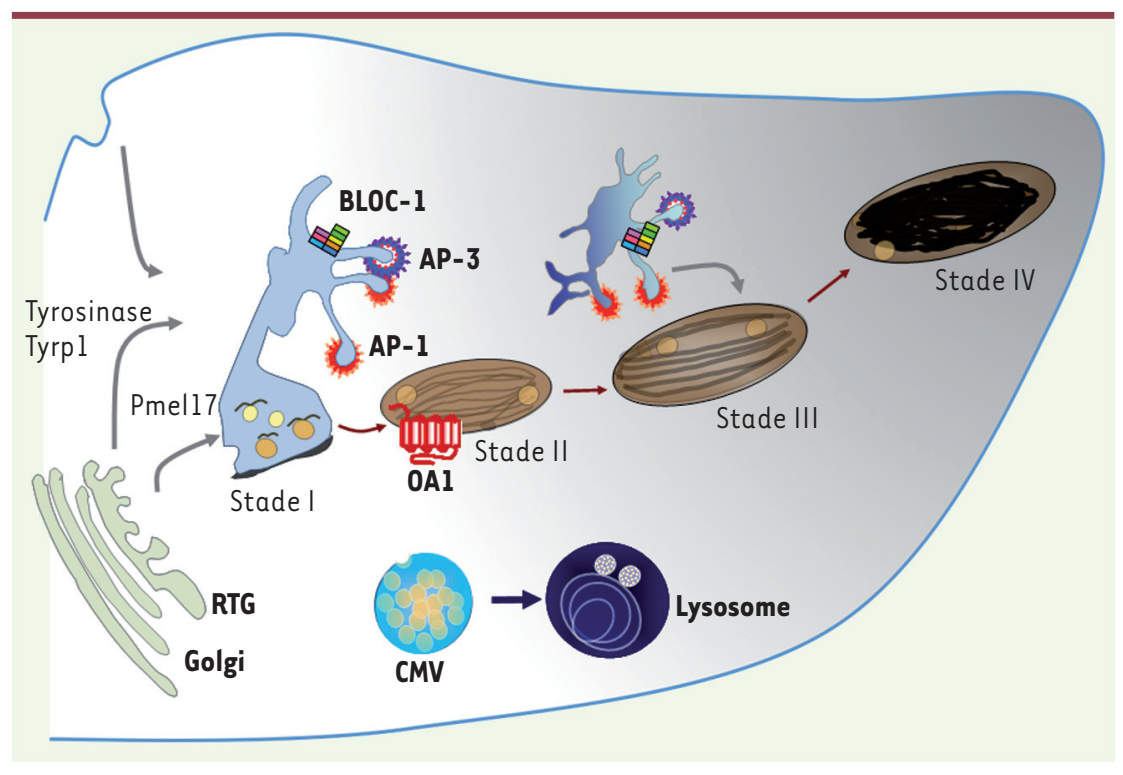

Figure 6. Spécialisation du trafic intracellulaire dans le mélanocyte. Les adaptateurs $A P-1$ et AP-3 ainsi que les complexes BLOC régulent le transport des enzymes mélanosomales (tyrosinase et Tyrpl) des endosomes aux mélanosomes. Par ailleurs, l'adaptateur AP-1 est également impliqué dans le positionnement des endosomes à proximité des mélanosomes grâce son interaction avec un moteur moléculaire, la kinésine KIFl3A (non représentée ici). Ce positionnement facilite les échanges entre endosomes et mélanosomes requis pour la maturation de ce compartiment. $\mathrm{OAl}$ est un récepteur à 7 domaines transmembranaires, localisé dans les mélanosomes immatures, qui contrôle la formation des prémélanosomes.

cargos des endosomes aux mélanosomes. Dès lors, la génération d'intermédiaires de transport et la polarisation d'un tel trafic depuis le compartiment donneur vers l'accepteur requièrent une étroite coordination entre le tri moléculaire et le positionnement des organites (Figure 6).

Dans les mélanocytes, les mélanosomes les plus pigmentés (stades III et IV) se distribuent à la périphérie cellulaire. Cette particularité spatiale nécessite que les enzymes de la mélanogenèse soient transportées du centre de la cellule, où elles sont synthétisées, vers la périphérie, où elles agissent. Les moteurs moléculaires, de types myosines ou kinésines, s'associent respectivement au cytosquelette d'actine ou au réseau de microtubules et sont nécessaires à la formation des intermédiaires de transport (tubules et/ou vésicules) et à leur déplacement jusqu'au compartiment cible. Nos travaux récents montrent qu'une kinésine, KIFl3A, est nécessaire au transport de l'enzyme Tyrpl des endosomes aux mélanosomes. Ce moteur, via son interaction avec AP-1, coordonne à la fois le tri des enzymes dans les endosomes ainsi que le transport et le positionnement de ces derniers à proximité des mélanosomes [19]. De plus, le moteur KIF13A et l'adapteur AP-1 interagissent tous les deux avec le cargo Tyrpl. Dès lors, ces observations permettent de proposer un modèle selon lequel AP-1 et KIFl3A, en association avec $B L O C-1$ et $B L O C-2$, réguleraient le tri des enzymes au niveau des endosomes précoces puis leur transport au contact des mélanosomes.

\section{Comment les cargos sont-ils délivrés aux mélanosomes?}

L'une des dernières étapes permettant aux cargos de la mélanogenèse de rejoindre leur destination est la fusion des endosomes avec les mélanosomes en cours de maturation. Bien que très peu de données expérimentales permettent de comprendre ce processus, les observations en microscopie électronique mettent en évidence des continuités membranaires entre les endosomes et les mélanosomes [19].
Dans les mélanocytes, le trafic endosome-mélanosome semble s'effectuer par l'intermédiaire de tubules membranaires [19]. Ces observations ne permettent pas de discriminer la nature antérograde ou rétrograde du transport qui s'opère via ces tubules membranaires et les deux possibilités ne sont pas mutuellement exclusives. Dès lors, le mélanosome poursuit vraisemblablement sa maturation grâce à la régulation de l'équilibre entre l'apport et l'export des protéines qui le composent.

Dans les cellules eucaryotes, le processus de fusion membranaire est régi par une famille de protéines appelées SNARE définies comme la machinerie moléculaire minimale responsable de la fusion des membranes biologiques [29]. Actuellement, aucune d'entre elles n'a été directement impliquée dans ce processus au cours de la mélanogenèse. Cependant plusieurs SNARE de la voie d'endocytose sont présentes à des taux élevés dans les mélanocytes [30] et l'une d'entre elles, la syntaxine-13, interagit directement avec BLOC-1 ou le complexe HOPS (homotypic protein sorting) [31]. Cette SNARE est localisée dans les endosomes de recyclage [32] et apparaît comme un bon candidat à la régulation de la fusion de ce compartiment avec les mélanosomes. Par ailleurs, la distribution de plusieurs SNARE endocytiques est altérée dans les mélanocytes déficients pour AP-3 ou BLOC-1, suggérant que ces complexes puissent moduler, directement ou non, l'activité de ces SNARE [23, 25]. Cependant, identifier les SNARE qui participent à décrypter la fusion endosomes-mélanosomes, leur régulation et le rôle des protéines mutées dans le HPS restent des objectifs qui méritent d'être abordés. 


\section{Les gènes de l'albinisme et leur rôle dans la mélanogenèse}

Bien que l'analyse des modèles de HPS nous ait appris que la progression des événements au cours de la mélanogenèse est largement régulée par des mécanismes ubiquitaires du trafic intracellulaire, les étapes de biosynthèse du pigment nécessitent un niveau supplémentaire de spécialisation. Ces étapes sont réalisées par des protéines exprimées spécifiquement par les mélanocytes et dont les gènes sont mutés dans des pathologies de la pigmentation couramment appelées albinismes. L'albinisme regroupe un ensemble hétérogène de troubles héréditaires résultant en une hypopigmentation variable de la peau, de l'iris et de la rétine, caractérisée par des défauts du nombre, de la structure et/ou de la fonction des mélanosomes. En outre, l'albinisme peut être associé à des défauts d'autres types cellulaires non pigmentés qui produisent des LRO, comme dans le HPS et le syndrome de Chediak-Higashi (CH) [33]. Toutefois, l'albinisme primaire est exclusivement dû aux mutations d'au moins un des cinq gènes codant pour des protéines transmembranaires du mélanosome dont les fonctions sont essentielles à la synthèse du pigment et à la maturation du mélanosome: tyrosinase, Tyrpl, P (pink eyed dilution), MATP (membrane-associated transporter protein)/SLC45A2 et OAl (ocular albinism 1)/GPRI43 (G protein-coupled receptor 143) (voir Encadré 3).

\section{OAl, le garant de l'identité du mélanosome?}

Le gène codant pour $\mathrm{OAl}$, dont l'expression est restreinte aux cellules pigmentées de la peau et des yeux, a été identifié depuis plus de 10 ans [34], mais sa fonction n'est toujours pas complètement comprise [35]. OAl est une glycoprotéine à sept domaines transmembranaires qui présentent peu d'homologies de séquence mais d'importantes similitudes structurelles et fonctionnelles avec les récep-

\section{Les albinismes oculocutanés}

Les mutations de la tyrosinase et de Tyrpl sont responsables des albinismes oculocutanés 1 et 3 (ocular cutaneous albinism type 1 et type 3 , OCAl et OCA3) caractérisés respectivement par une absence de pigment et une réduction de la quantité de mélanine [49]. P (pink eyed dilution) et MATP (membrane-associated transporter protein) sont deux protéines canaux qui contrôlent le pH ou l'osmolarité des mélanosomes. Leurs mutations sont la cause des albinismes oculocutanés (OCA2 et OCA4) caractérisés par une diminution de la quantité de mélanine. La mutation du gène $\mathrm{OAl}$ est responsable d'une forme particulière d'albinisme oculaire (ocular albinism type 1) qui, contrairement à ce qui est observé pour les OCA, n'affecte pas directement la production du pigment mais a pour conséquence une diminution du nombre des mélanosomes [38]. L'une des caractéristiques de cette maladie (chez les patients affectés ou dans des modèles murins) est la présence, dans les cellules pigmentées de la rétine et de la peau, de mélanosomes géants (macromélanosomes) [50]. Ainsi, OAl pourrait contrôler la mélanogenèse via la régulation du nombre et de la taille des mélanosomes. teurs couplés aux protéines G (GPCR, G protein coupled receptor) [36]. Les GPCR se localisent à la membrane plasmique et possèdent un domaine de liaison à un ligand extracellulaire qui peut être une hormone ou un neurotransmetteur. La liaison de ce ligand induit un changement de conformation du récepteur qui interagit avec des GTPases intracellulaires ou protéines $G$, nommées Gi, Go ou Gq. Celles-ci modulent l'activité du GPCR et lui permettent d'envoyer des signaux intracellulaires en réponse aux stimulus physiologiques. À l'instar des GPCR, OAl lie un ligand, la L-DOPA (un produit intermédiaire de la voie de biosynthèse de la mélanine) [37], et interagit avec les protéines $G$ de type inhibiteur (Gi) [35]. Cependant, dans les mélanocytes humains de la peau, OAl s'associe majoritairement à la membrane des lysosomes et des mélanosomes immatures (stades I et II) [38]. Sa distribution intracellulaire est due à deux motifs protéiques responsables de son tri, dont les mutations altèrent son trafic intracellulaire et sa fonction [40]. Dès lors, la localisation d'OAl aux membranes intracellulaires ainsi que sa topologie membranaire suggèrent que sa liaison à la L-DOPA intervient dans la lumière du mélanosome. Ainsi, OAl pourrait transduire un signal de l'intérieur du compartiment vers le cytosol.

Les pathologies associées à l'albinisme oculaire de type 1 suggèrent que $O A l$ joue un rôle-clé dans la maturation des mélanosomes. En effet, par l'intermédiaire d'une cascade de transduction dépendante de la protéine Gai3, OAl régulerait la biogenèse des mélanosomes. Des souris chez lesquelles l'expression de Gai3 est éteinte ont des phénotypes semblables à ceux observés dans l'albinisme oculaire de type 1 [40]. Récemment, il a été montré qu'OAl participe aux étapes précoces de la mélanogenèse en contrôlant la composition protéique et l'identité des prémélanosomes [38]. Par exemple, en l'absence d'OAl, les mélanocytes produisent des prémélanosomes anormaux qui accumulent à la fois les protéines de la prémélanogenèse (Pmel17) et celles de la mélanogenèse tardive (Tyrp1). Ces observations suggèrent que la ségrégation et/ou le tri de ces protéines sont affectés [38]. Dès lors, $0 \mathrm{Al}$ modulerait le trafic des protéines de la mélanogenèse en régulant négativement la maturation des mélanosomes [38]. L'étude de la fonction des protéines mutées dans l'albinisme facilite la compréhension de la physiopathologie liée à ce trouble. Cette étude permet également la mise en évidence de nouvelles voies de signalisation et de régulation du trafic intracellulaire des cellules spécialisées dans la formation d'organites spécifiques, dont les mélanosomes font partie. 


\section{Le transfert des mélanosomes : l'ultime étape?}

Dans l'épiderme, le transfert des mélanosomes des mélanocytes aux kératinocytes est nécessaire à la pigmentation de la peau et à sa protection lors de son exposition aux rayons UV. Un mélanocyte de l'épiderme, grâce à ses projections dendritiques, peut contacter environ quarante kératinocytes [41]. L'interaction entre ces deux types cellulaires met en cause de multiples protéines d'adhésion créant un contact intime qui, par analogie aux synapses neuronales ou immunologiques, est appelé synapse pigmentaire [42]. L'une des étapes précédant le transfert est la mobilisation des mélanosomes sous la membrane plasmique, aux extrémités dendritiques. Les mélanosomes y sont transportés via la kinésine-1 et le réseau de microtubules [43]. Une fois en périphérie, les mélanosomes s'associent au réseau d'actine sous-membranaire via le complexe tripartite Rab27a-mélanophiline-myosine $\mathrm{Va}$ [44]. Le rôle de ce complexe dans le transport et l'arrimage des mélanosomes à la périphérie des mélanocytes a été mis en évidence grâce à l'étude de mélanocytes issus de patients et de souris modèles d'une autre maladie lysosomale, le syndrome de Griscelli [45]. Les mécanismes impliqués dans le transfert des mélanosomes depuis les mélanocytes aux kératinocytes restent encore aujourd'hui mal compris. Trois processus distincts mais non mutuellement exclusifs sont proposés pour expliquer l'acquisition du pigment par les kératinocytes: (1) l'exocytose du pigment nu de toute membrane suivie de son endocytose par le kératinocyte ; (2) le transfert des mélanosomes par des nanotubes établis entre mélanocytes et kératinocytes; (3) la phagocytose par le kératinocyte des extrémités dendritiques des mélanocytes riches en mélanosomes [42]. Cependant, gageons que si autant d'efforts sont entrepris pour comprendre ce transfert que pour élucider la mélanogenèse, ce processus-clé de la pigmentation devrait livrer rapidement ses secrets.

\section{Conclusion}

Au cours de ces dernières années, les bases nécessaires à la compréhension des mécanismes et machineries cellulaires intervenant dans la formation des mélanosomes ont été élucidées. La pertinence de l'étude des mécanismes de biogenèse du mélanosome est intimement liée à l'importance de la compréhension du maintien de l'état pigmenté de l'épiderme, non seulement d'un point de vue cosmétique, mais surtout parce qu'il est essentiel à la photoprotection contre les radiations des rayons UV (auxquels l'être humain est exposé). L'incidence des cancers de la peau, dont en particulier le mélanome, est inversement proportionnel à l'état de pigmentation d'un épiderme. De plus, le risque de développer un mélanome dépend du patrimoine génétique de chacun. $\varepsilon n$ effet, dans certaines familles, l'expression de variants génétiques augmente la susceptibilité à ce cancer. Beaucoup, parmi les variants des gènes déjà identifiés, codent pour des protéines mélanosomales et pour des protéines impliquées dans la biogenèse des mélanosomes [46]. II serait donc essentiel de comprendre comment l'expression de ces variants affecte le processus de formation du mélanosome. D'autre part, un grand nombre de protéines impliquées dans le trafic intracellulaire sont codées par des gènes dont les mutations sont à l'origine de maladies génétiques. Les syndromes de Griscelli, de Chediak-Higashi et d'Hermansky-Pudlak, qui affectent la pigmentation, en sont les meilleurs exemples. Cependant, ces maladies génétiques dites lysosomales altèrent la formation et la fonction de nombreux LRO, avec pour conséquence de multiples troubles comme un défaut de coagulation, des fibroses pulmonaires ou des déficits immunitaires. Ces multiples effets démontrent que la biogenèse des différents LRO emprunte en partie les mêmes mécanismes moléculaires. Ainsi, les données déjà acquises sur la formation des mélanosomes faciliteront sans aucun doute la compréhension des mécanismes de biogenèse de cette famille de compartiments membranaires et des troubles qui leur sont associés. $\diamond$

\section{SUMMARY}

Biogenesis of melanosomes:

the chessboard of pigmentation

Melanosomes are lysosome-related organelles in retinal pigment epithelial cells and epidermal melanocytes in which melanin pigments are synthesized and stored. Melanosomes are generated by multistep processes in which an immature unpigmented organelle forms and then subsequently matures. Such maturation requires inter-organellar transport of protein cargos required for pigment synthesis but also recruitment of effector proteins necessary for the correct transport of melanosomes to the cell periphery. Several studies have started to unravel the main pathways and mechanisms exploited by melanosomal proteins involved in melanosome structure and melanin synthesis. A major unexpected finding seen early in melanosome biogenesis showed the similarities between the fibrillar sheets of premelanosomes and amyloid fibrils. Late steps of melanosome formation are dependent on pathways regulated by proteins encoded by genes mutated in genetic diseases such as the Hermansky-Pudlak Syndrom (HPS) and different types of albinism. Altogether the findings from the past recent years have started to unravel how specialized cells integrate unique and ubiquitous molecular mechanisms in subverting the endosomal system to generate cell-type specific structures and their associated functions. Further dissection of the melanosomal system will likely shed light not only on the biogenesis of lysosome-related organelles but also on general aspects of vesicular transport in the endosomal system. $\diamond$

\section{REMERCIEMENTS}

Nous tenons à remercier nos collègues Danièle Tenza, Ilse Hurbain, Maryse Romao, Sabrina Simoes et Leila Rochin pour leur contribution à la résolution de l'énigme de la mélanogenèse. Merci également à Michael Marks (University of Pennsylvania) 
pour la collaboration fructueuse outre-atlantique et à Fabio Benedetti pour sa participation à cette synthèse. Ce travail a été rendu possible grâce au soutien de la Fondation pour la recherche médicale (FRM), L'Association pour la recherche sur le cancer (ARC), les National institutes of health (NIH), le CNRS, l'Inserm et l'Institut Curie.

\section{CONFLIT D'INTÉRÊTS}

Les auteurs déclarent n'avoir aucun conflit d'intérêts concernant les données publiées dans cet article.

\section{RÉFÉRENCES}

1. Ebanks JP, Wickett RR, Boissy RE. Mechanisms regulating skin pigmentation: the rise and fall of complexion coloration. Int J Mol Sci 2009; $10:$ 4066-87.

2. Seiji M, Fitzpatrick TM, Simpson RT, Birbeck MSC. Chemical composition and terminology of specialized organelles (melanosomes and melanin granules) in mammalian melanocytes. Nature $1963 ; 197: 1082-4$.

3. Raposo G, Tenza D, Murphy DM, et al. Distinct protein sorting and localization to premelanosomes, melanosomes, and lysosomes in pigmented melanocytic cells. J Cell Biol $2001 ; 152: 809-24$.

4. Raposo G, Marks MS. Melanosomes--dark organelles enlighten endosomal membrane transport. Nat Rev Mol Cell Biol 2007 ; 8 : 786-97.

5. Kushimoto T, Basrur V, Valencia J, et al. A model for melanosome biogenesis based on the purification and analysis of early melanosomes. Proc Natl Acad Sci USA $2001 ; 98$ : 10698-703.

6. Yasumoto $\mathrm{K}$, Watabe $\mathrm{H}$, Valencia JC, et al. Epitope mapping of the melanosomal matrix protein gp100 (PMEL17): rapid processing in the endoplasmic reticulum and glycosylation in the early Golgi compartment. J Biol Chem $2004 ; 279$ : 28330-8.

7. Berson JF, Harper DC, Tenza D, et al. Pmell7 initiates premelanosome morphogenesis within multivesicular bodies. Mol Biol Cell $2001 ; 12: 3451-64$.

8. Gould GW, Lippincott-Schwartz J. New roles for endosomes: from vesicular carriers to multipurpose platforms. Nat Rev Mol Cell Biol 2009 ; $10: 287-92$.

9. Simons M, Raposo G. Exosomes-vesicular carriers for intercellular communication. Curr Opin Cell Biol 2009; $21: 575-81$.

10. Fowler DM, Koulov AV, Alory-Jost C, et al. Functional amyloid formation within mammalian tissue. PLoS Biol $2006 ; 4$ : e6.

11. Watt $B$, van Niel G, Fowler DM, et al. N-terminal domains elicit formation of functional Pmell7 amyloid fibrils. J Biol Chem 2009 ; 284 : 35543-55.

12. Berson JF, Theos AC, Harper DC, et al. Proprotein convertase cleavage liberates a fibrillogenic fragment of a resident glycoprotein to initiate melanosome biogenesis. J Cell Biol 2003 ; $161: 521-33$

13. Hurbain I, Geerts WJ, Boudier T, et al. Electron tomography of early melanosomes: implications for melanogenesis and the generation of fibrillar amyloid sheets. Proc Natl Acad Sci USA 2008 ; 105 : 19726-31.

14. Theos AC, Truschel ST, Tenza D, et al. A lumenal domain-dependent pathway for sorting to intralumenal vesicles of multivesicular endosomes involved in organelle morphogenesis. Dev Cell $2006 ; 10: 343-54$

15. Roxrud I, Stenmark H, Malerod L. ESCRT and Co. Biol Cell 2010 ; 102 : 293-318.

16. Wei ML. Hermansky-Pudlak syndrome: a disease of protein trafficking and organelle function. Pigment Cell Res $2006 ; 19: 19-42$

17. Theos AC, Tenza D, Martina JA, et al. Functions of AP-3 and AP-1 in Tyrosinase Sorting from Endosomes to Melanosomes. Mol Biol Cell 2005 ; 16 : 5356-72.

18. Gautam R, Novak EK, Tan J, et al. Interaction of Hermansky-Pudlak Syndrome genes in the regulation of lysosome-related organelles. Traffic $2006 ; 7: 779-92$.

19. Delevoye C, Hurbain I, Tenza D, et al. AP-1 and KIF13A coordinate endosomal sorting and positioning during melanosome biogenesis. J Cell Biol 2009; 187 : 247-64.

20. Bonifacino JS, Traub LM. Signals for sorting of transmembrane proteins to endosomes and lysosomes. Annu Rev Biochem 2003 ; 72 : 395-447.

21. Oetting WS, Fryer JP, Shriram S, King RA. Oculocutaneous albinism type 1 : the last 100 years. Pigment Cell Res $2003 ; 16$ : 307-11.

22. Setty SR, Tenza D, Sviderskaya EV, et al. Cell-specific ATP7A transport sustains copper-dependent tyrosinase activity in melanosomes. Nature 2008 ; 454 : 1142-6.

23. Setty SR, Tenza D, Truschel ST, et al. BLOC-1 is required for cargo-specific sorting from vacuolar early endosomes toward lysosome-related organelles. Mol Biol Cell $2007 ; 18$ : 768-80.

24. Di Pietro SM, Falcon-Perez JM, Tenza D, et al. BLOC-1 interacts with BLOC-2 and the AP- 3 complex to facilitate protein trafficking on endosomes. Mol Biol Cell $2006 ; 17: 4027-38$.

25. Salazar G, Craige B, Styers ML, et al. BLOC-1 complex deficiency alters the targeting of adaptor protein complex-3 cargoes. Mol Biol Cell $2006 ; 17: 4014-26$.

26. Di Pietro SM, Falcon-Perez JM, Dell'Angelica EC. Characterization of BLOC-2, a complex containing the Hermansky-Pudlak syndrome proteins HPS3, HPS5 and HPS6. Traffic $2004 ; 5$ : 276-83.
27. Gautam R, Chintala S, Li W, et al. The Hermansky-Pudlak syndrome 3 (cocoa) protein is a component of the biogenesis of lysosome-related organelles complex-2 (BLOC-2). J Biol Chem 2004 ; 279 : 12935-42.

28. Boissy RE, Richmond B, Huizing M, et al. Melanocyte-specific proteins are aberrantly trafficked in melanocytes of Hermansky-Pudlak syndrome-type 3. Am J Pathol $2005 ; 166: 231-40$

29. Martens S, McMahon HT. Mechanisms of membrane fusion: disparate players and common principles. Nat Rev Mol Cell Biol 2008 ; 9 : 543-56.

30. Wade N, Bryant NJ, Connolly LM, et al. Syntaxin 7 complexes with mous Vps10p tail interactor $1 b$, Syntaxin 6, vesicle-associated membrane protein (VAMP)8, and VAMP7 in B16 melanoma cells. J Biol Chem 2001 ; $276: 19820-7$.

31. Huang L, Kuo YM, Gitschier J. The pallid gene encodes a novel, syntaxin 13 -interacting protein involved in platelet storage pool deficiency. Nat Genet $1999 ; 23: 329-32$.

32. Prekeris R, Klumperman J, Chen YA, Scheller RH. Syntaxin 13 mediates cycling of plasma membrane proteins via tubulovesicular recycling endosomes. J Cell Biol 1998 ; 143 : 957-71.

33. Dell'Angelica EC, Aguilar RC, Wolins N, et al. Molecular characterization of the protein encoded by the Hermansky-Pudlak syndrome type 1 gene. J Biol Chem $2000 ; 275: 1300-6$.

34. Bassi MT, Bergen AA, Bitoun P, et al. Diverse prevalence of large deletions within the $0 \mathrm{Al}$ gene in ocular albinism type 1 patients from Europe and North America. Hum Genet $2001 ; 108: 51-4$

35. Schiaffino MV. Signaling pathways in melanosome biogenesis and pathology. Int J Biochem Cell Biol $2010 ; 42$ : 1094-104.

36. Innamorati G, Piccirillo R, Bagnato P, et al. The melanosomal/lysosomal protein $\mathrm{OAl}$ has properties of a $\mathrm{G}$ protein-coupled receptor. Pigment Cell Res $2006 ; 19: 125-35$

37. Lopez VM, Decatur CL, Stamer WD, Lynch RM, McKay BS. L-DOPA is an endogenous ligand for OAl. PLoS Biol $2008 ; 6$ : e236.

38. Giordano F, Bonetti C, Surace EM, et al. The ocular albinism type 1 (OAl) G-protein-coupled receptor functions with MART-l at early stages of melanogenesis to control melanosome identity and composition. Hum Mol Genet $2009 ; 18: 4530-45$

39. Piccirillo R, Palmisano I, Innamorati G, et al. An unconventional dileucinebased motif and a novel cytosolic motif are required for the lysosomal and melanosomal targeting of OAl. J Cell Sci 2006; 119:2003-14.

40. Young $A$, Powelson $\varepsilon B$, Whitney $I \varepsilon$, et al. Involvement of $O A l$, an intracellula $G P C R$, and $G$ alpha i3, its binding protein, in melanosomal biogenesis and optic pathway formation. Invest Ophthalmol Vis Sci $2008 ; 49: 3245-52$.

41. Fitzpatrick TB, Breathnach AS. The epidermal melanin unit system. Dermatol Wochenschr $1963 ; 147:$ 481-9.

42. Van Den Bossche K, Naeyaert JM, Lambert J. The quest for the mechanism of melanin transfer. Traffic $2006 ; 7: 769-78$.

43. Hara M, Yaar M, Byers HR, et al. Kinesin participates in melanosomal movement along melanocyte dendrites. J Invest Dermatol 2000 ; $114: 438-43$.

44. Seabra MC, Coudrier $\varepsilon$. Rab GTPases and myosin motors in organelle motility. Traffic $2004 ; 5: 393-9$

45. Bahadoran P, Ortonne J, Ballotti R. Que trafiquent les mélanosomes? Med Sci (Paris) $2002 ; 18: 205-9$.

46. Gudbjartsson DF, Sulem P, Stacey SN, et al. ASIP and TYR pigmentation variants associate with cutaneous melanoma and basal cell carcinoma. Nat Genet $2008 ; 40: 886-91$

47. Kummer MP, Maruyama H, Huelsmann C, et al. Formation of Pmell7 amyloid is regulated by juxtamembrane metalloproteinase cleavage, and the resulting C-terminal fragment is a substrate for gamma-secretase.J Biol Chem $2009 ; 284: 2296-306$.

48. Ehehalt R, Keller P, Haass C, et al. Amyloidogenic processing of the Alzheimer beta-amyloid precursor protein depends on lipid rafts. J Cell Biol 2003; $160: 113-23$

49. Yamaguchi Y, Brenner M, Hearing VJ. The regulation of skin pigmentation. J Biol Chem 2007 ; 282 : 27557-61.

50. Incerti B, Cortese K, Pizzigoni A, et al. Oal knock-out: new insights on the pathogenesis of ocular albinism type 1 . Hum Mol Genet $2000 ; 9: 2781-8$.

\section{TIRÉS À PART}

G. Raposo 